\title{
Éléments pour une anthropologie de la présence
}

Materials for an anthropology of presence

\section{Francis Affergan}

\section{OpenEdition}

Journals

Édition électronique

URL : https://journals.openedition.org/ress/3222

DOI : $10.4000 /$ ress.3222

ISBN : 1663-4446

ISSN : 1663-4446

Éditeur

Librairie Droz

Édition imprimée

Date de publication : 15 novembre 2015

Pagination : 17-49

ISSN : 0048-8046

Référence électronique

Francis Affergan, «Éléments pour une anthropologie de la présence », Revue européenne des sciences sociales [En ligne], 53-2 | 2015, mis en ligne le 15 novembre 2018, consulté le 21 septembre 2021. URL : http://journals.openedition.org/ress/3222 ; DOI : https://doi.org/10.4000/ress.3222 


\title{
ÉLÉMENTS POUR UNE ANTHROPOLOGIE DE LA PRÉSENCE
}

\author{
FRANCIS AFFERGAN \\ Université Paris Descartes, CANTHEL \\ nagreffa@aol.com
}

\begin{abstract}
Résumé. À partir des recherches ethnographiques sur certaines sociétés et cultures tout autour de la planète, je plaide pour la construction d'une anthropologie de la présence. Chez les Samoans, les habitants de la Lucanie d'Italie du Sud, les Runa d'Amazonie occidentale et les Korowaï de Papouasie Nouvelle Guinée, l'anthropologie travaille sur des choses et des objets dans leur manifestation même et selon les modalités de leur apparition. J'étudie certaines thématiques pour nourrir mes hypothèses: la quotidienneté et l'ordinaire de la vie, la spatialisation de la chair, la facticité des corps et des actions en l'absence d'un cadre symbolique, etc. Je m'emploie à expliquer si et comment une chose peut avoir un sens sans une intention symbolique. Qu'est-ce qu'un monde dans sa présence même sans un arrière-monde? Comment la présence des choses confère une signification sans recours à la référence? Cependant, ces sociétés nous apprennent aussi que la présence s'obtient par le déchirement et la division. C'est vers une anthropologie de l'entrelacement des regards et des corps, des sensations et des sentiments que nous tournerons notre attention scientifique.
\end{abstract}

Mots-clés: aliénation, chair, corps, espace, présence, sensation, symbolique, temps.

\begin{abstract}
From research about societies and cultures through all over the world, I plead for the construction of an anthropology of the presence. Among the Samoans, the inhabitants of Lucanie (South Italy), the Runa (Amazonie) and the Korowaï (Papua New Guinea), anthropology works on things and objects in their manifestation and in accordance with their appearance. I study some topics in order to sustain my hypothesis: the everyday nature, the ordinary of the life, the spatialization of the flesh, the artifice of the bodies and the actions without the symbolic frame, etc. I try to explain if and how a thing can have a meaning without a symbolic intention. What is a world in his presence without a background? How the presence of things gives a meaning without resort to reference? However, these societies teach us as well that the presence is obtained by tearing apart and division. It's toward an anthropology of intertwining of glances and bodies, of sensations and feelings that I turn my scientific attention.
\end{abstract}

Keywords: alienation, body, flesh, presence, sensation, space, symbolic, time. 
Une anthropologie réaliste consisterait à étudier les choses et les objets sous l'angle de la présence de leur manifestation, selon les modalités de leur apparition sensible. Or toute présence d'un monde implique une fragilité existentielle, à savoir celle de la perte, de l'abandon et de l'angoisse. Nous partirons donc de l'hypothèse ontologique selon laquelle les choses sont ce qu'elles sont non seulement en raison de leur identité propre, mais aussi et surtout, parce qu'elles rendent visibles les autres choses, sans pour autant s'assimiler à elles, cependant toujours avec le risque d'une déroute liée à un brouillage de leur eccéité (c'est-à-dire le fait d'être là, posé dans le présent).

Les symptômes sociaux et culturels, ce sur quoi nous travaillons dans un monde visible, loin de n'être que les signes d'un autre monde, seraient à examiner comme la structure fondamentale de l'expérience existentielle elle-même. En cela la présence confère un sens (Sinn: sens intentionnel) sans avoir recours à une référence (Bedeutung: extension). Prier, invoquer le royaume des morts, intercéder, intervenir dans la nature sont aussi des choses sociales et culturelles que les agents fabriquent dans l'être-là d'un monde fini, contingent et immanent.

Toute présence s'obtiendrait donc sur le fond du déchirement et de la division, puisque la présence, dans sa manifestation même, n’est jamais égale à elle-même. D’une part, les choses sont ce qu'elles sont, et d'autre part, elles ne sont jamais ce qu'elles sont, mais toujours autre chose, en avant, à côté, en arrière d'elles-mêmes, creusées par leur manque à dire.

C'est cette crise que nous aimerions interroger. Car il est coutumier d'affirmer que l'activité symbolique qui caractérise toute société humaine repose sur le passage du continu (qui n’a pas de sens puisque la réalité est toujours réductible à la contiguïté des choses qui se succèdent) au discontinu (les procédures de découpage de la réalité en catégories intelligibles). Nous nous efforcerons de montrer que le continu de la vie, avant même sa symbolisation, a déjà un sens, comme par exemple l'étude du corps propre nous y invite, dans la mesure où tout corps senti (le mien ou celui d'un autre) suppose un corps sentant (le mien ou celui d'un autre), avant même la confection de catégories susceptibles de rendre compte de leur liaison. 
Certes, le monde ne peut être perçu que s'il existe posé dans son objectivité. Mais, pour moi, je n'existe à mon tour que pour autant qu'il existe un monde dans lequel je suis situé en tant que corps, chair, souffrance et vécu intérieur de mes sens.

\section{DE LA PRÉSENCE}

Walter Benjamin (1991 [1940]) propose une étrange conception de l'histoire. Selon lui, elle n'aurait de sens et d'intérêt qu'en tant que suite ou exposition de tableaux événementiels, mais non nécessairement liés dans une série. L'histoire nous ferait voir, nous apprendrait à voir des événements, mais en l'absence de toute représentation. Cette théorie s’appuie sur une survalorisation de la présence, due à l'impossibilité de prendre conscience de l'événement au moment même où il a lieu.

Il est vrai que sa soudaineté brutale étouffe toute capacité à le restituer dans la simultanéité. Bien que nous soyons présent à lui, il nous échappe et se métamorphose en trace que l'écriture aura pour tâche d'évoquer ou d'invoquer. L'événement, en tant que saut, contraint l'historien ou l'anthropologue, présent mais s'effaçant dans cette présence même, à revenir, tel un fantôme, sur la scène pour le revisiter par l'écriture. Cette présence en éclipse s'explique, pour Benjamin, par la double qualité du temps : il passe une première fois et revient ensuite sous la forme élaborée de la narration symbolique ${ }^{\mathrm{I}}$.

Ce monde de l'être-là, pour lequel militait Benjamin, dans sa version historique qui ne fut pas comprise de son temps, on le retrouve chez Ernesto De Martino dans sa version ethnologique et anthropologique. Les expériences culturelles par lesquelles les humains construisent leur identité, comme les cérémonies magiques ou religieuses, comme la transe ou le sacrifice, indiquent à la fois un ancrage existentiel et une fragilité ontologique que De Martino appelle «crise de la présence ». Au cours de ces expériences exis-

I «Articuler historiquement le passé signifie: discerner ce qui, dans ce passé même, sous la constellation d'un seul et même instant, le rassemble. C'est dans l'instant historique et uniquement en lui, qu'est seulement possible la connaissance historique. Mais cette connaissance dans l'instant historique est toujours elle-même la connaissance d'un instant» (Benjamin, 1991 [1940], p.348). 
tentielles, dans lesquelles la culture se vit sur le mode d'une subjectivation charnelle, il y a un risque de perte de soi, de disparition du corps propre, tant les limites de la vie et de la mort sont à ce point frôlées, voire transgressées : «La présence personnelle de soi-même, l' "être-là”, l'âme: elle "fuit" son siège, elle peut être "ravie”, “dérobée”, “mangée”, etc. ; c'est un oiseau, un papillon, un souffle» (De Martino, 1999 [1948], p. 98).

La présence devient une catégorie complexe et contradictoire dans la mesure où elle symptomatise l'enracinement de l'existence par le corps et où, simultanément, elle en indique la déficience puisqu'elle risque toujours de se perdre (ibid., p.99). À la fois avoir et être, cette présence joue de la confusion entre la rétention et la néantisation. Les expériences culturelles sont bien des événements dangereux dès l'instant où, à chaque fois, ils remettent en jeu l'existence elle-même dans le risque permanent qu'elle encourt : celui de disparaître ou de continuer à vivre dans l'angoisse de la mort ou de la déformation de soi. La présence, «risquant toujours de s'égarer», et ainsi de ne pouvoir se maintenir comme présence (ibid., p. I02), tente alors de se racheter par une représentation de l'objet magique ou sacré qui fascine et paralyse.

Cette représentation, nous pouvons l'appeler symbolique dans la mesure où elle se met à la place de la présence charnelle et temporelle, permettant ainsi de comprendre par objectivation interposée, mais au prix d'une perte ou d'un amoindrissement. C’est la raison pour laquelle cette présence peut être vécue comme un «péché» (ibid., p. 205), dès lors que toute expérience culturelle est limitée par les normes de l'histoire et des civilisations. Les expériences culturelles en appellent au symbolique au sens où, pour exister et pour représenter un monde, elles se trouvent contraintes de passer par une phase de «castration » de la présence spatio-temporelle de la chose. Une couche de sur-sens vient ainsi se superposer à un premier bâti pourvoyeur d'un sens intentionnel que Frege appelait Sinn sans nécessiter une signification supplémentaire extensive et référentielle (Bedeutung au sens de Frege). De Martino l'explicite en reprenant l'exemple du monde magique: «l'intérêt dominant du monde magique n’est pas de réaliser des formes particulières de la vie spirituelle, mais de conquérir et de consolider l'être au monde élémentaire, ou présence, de la personne» (ibid., p. 208). 
C'est en cela qu'à l'instar du masque, l'événement magique doit servir, être utile à, permettre d'exister dans une temporalité plus accordée, une forme de vie en accord quasi musical avec un monde. Or cette présence est indétachable du risque qu'elle comporte en raison de sa possible disparition à tout moment. Il s'agit bien d'une double présence : celle de la chose ou de l'objet et celle de la personne qui s'y identifie. Et ce n'est pas tant la perte d'un sens qu'il s'agit de conjurer, que la perte d'un monde, celui habité par la personne qui fait l'expérience charnelle de l'action magique, c'est-à-dire qui est en train de devenir quelqu'un d'autre, à travers la sensibilité de son corps.

On est en mesure d'avancer maintenant une hypothèse, dans le sillon de Benjamin et de De Martino: l'événement culturel, quel qu'il soit, et en tant qu'expérience existentielle, est «un moment négatif» (ibid., p. 280), puisqu'il n'éprouve nul besoin de se hisser à la hauteur trompeuse du symbolique pour dégager un sens pratique. De Martino est même plus radical : la magie apparaît comme une «non-culture», s'émancipant ainsi de la structure sémantique de son message pour s'imposer comme le «drame d’une présence exposée au risque de ne pas être là ». En quelque sorte, la présence est toujours un moment catastrophique de l'existence car je peux en mourir. L’expérience magique ou la danse masquée conduisent le sujet à accepter la menace de la mort. La norme sociale, culturelle et civilisationnelle, et c'est ce en quoi elle relève du symbolique, permet seulement de la conjurer, dans un après-coup du sens.

Dans sa monographie sur les Korowai, Rupert Stasch montre en quoi et comment cette société du plateau central de la Papouasie occidentale a développé une forme de vie et des jeux de langage ancrés dans une physique et une métaphysique de la présence. Et le premier symptôme de cette présence est palpable dans la «résidence», catégorie devant être entendue comme «la question de savoir où le peuple se situe physiquement sur la terre »² (Stasch, 2009, p. 32). 
Cette présence dans la terre, à laquelle les Korowai tiennent comme à un ancrage ontique et ontologique, et par laquelle ils promulguent (enact) leur modalité relationnelle de la possession et de la propriété (ownership relation), loin de ne signifier qu’un attachement égoïste à une propriété privée, est dans le même temps leur façon de pratiquer l'unité humaine de la terre («Presence on land is a practice of human-land unity» [ibid., p. 33]). C'est cette coappartenance qui devient ainsi l'emblème, à leurs yeux, de cette relation de possession. C'est la raison pour laquelle l'humanité n'est pas divisée en deux parties inconciliables qui seraient les propriétaires de la terre, d'une part, et ceux qui y résident sans la détenir, d'autre part. C'est la même humanité qui se lie à elle-même par le truchement de la terre, qui devient dès lors un bien commun. Les différents niveaux (levels) de coappartenance entre ceux qui détiennent la terre sans l'habiter et ceux qui y résident sans la posséder sont d'une grande complexité, mais reposent en fait sur la seule notion de présence (ibid., p. 33) qui garantit et cautionne, physiquement et discursivement, le sentiment d’appartenance identitaire.

Cependant la présence ne se réduit pas, pour les Korowai, à l’actualité spatiotemporelle de la chose ou de la personne. La stratégie de la double désignation en témoigne. Chaque chose, animal, objet ou personne est désignée par paire de désignateurs. Ainsi un chien peut être appelé vipère de mort (death adder), un cochon un casoar (cassowary). Mais le nom substitutif porte une relation tellement obscure au référent qu'il est censé désigner qu’il ne doit jamais être prononcé en sa présence par crainte de le faire disparaître, ou de l'endommager (ibid., p. 89). Autrement dit, la relation entre les deux désignateurs n’appartient ni à l'ordre logique ni à l'ordre sémantique, ni à la logique du signifiant ni à celle du signifié, mais à une logique spatiale qui joue de la dialectique entre la présence et l'absence. Non seulement le deuxième désignateur, celui de substitution, ne renvoie pas à un référent circonscrit dans la réalité spatio-temporelle, ni directement au désignateur habituel, mais désigner ainsi peut tuer ou abîmer la chose si elle est présente. Si le chien entend «vipère de mort», il mourra. La présence du mot se substitue ainsi à la présence de la chose, manifestant une réalité qui fonctionne par paire mais sans investissement d'une quelconque logique sémantique. La seule logique qui semble compter pour les Korowai est 
celle d'une spatialité des choses dans la réalité sensible et des sons dans leur profération. C'est ce couple logique qui marque l'originalité de la pratique et des représentations de la pensée korowai. Si toutes les représentations, sensibles et discursives, fonctionnent par paire, ce n'est pas tant en raison d'un double monde, dont celui de l'arrière serait le symbole de celui de la réalité, qu'à cause d'une conception du langage qui se veut duelle et dont une partie ne peut se comprendre qu'en fonction de l'autre. Ainsi, pour désigner les relations entre les mondes visible et invisible, les Korowai ont-ils recours à la figure des «bûches croisées » (ibid., p. I02), ou bien pour évoquer les relations avec la belle-mère, utiliseront-ils la figure des «panneaux muraux». Cette logique peut être appelée figurative au sens où il s'agit de voir, comme à l'aide d'un schème, la chose, l'objet, le rapport ou la relation dont on veut parler.

Elle est figurative en cela qu'elle ne relève ni d'une logique sémantique ni d'une logique symbolique. En effet, la bûche croisée ne veut ni dire ni symboliser la dualité des mondes, mais seulement la montrer, l'exhiber comme dans un tableau. Je ne suis pas certain qu'il s'agisse d'une relation métonymique, comme semble le suggérer Stasch, dans la mesure où dans le dispositif métonymique, le lien entre les deux termes doit être nécessaire puisqu'aucune ressemblance n'est en mesure de les souder. Or, dans le langage korowai, il ne s'agit pas tant de nécessité que d'un accord sonore et figuratif, qui aurait pu être autre: «Une personne est mise pour et appelle la présence de l'unité de deux personnes comme paire, exactement au même titre que la paire se substitue à et appelle la présence d'une personne » (ibid.).

D’ailleurs, Stasch le reconnaît lui-même en parlant de «portraiturer» les relations à la belle-mère. Le présent ne se réduit donc pas à la présence pour les Korowai. En effet, il est à la fois connecté et séparé d’autres scènes qui n’ont pas de rapport direct avec lui. Le présent serait ce «mélange complexe de lien spatio-temporel et de disparité » (ibid., p. 270).

C'est la raison pour laquelle la présence comme face-à-face est insuffisante pour rendre compte de la complexité de la relation d'une personne à quelque chose d'autre. Plusieurs temporalités coexistent et cohabitent pour tenter de rendre compte d'un monde dans lequel «l'attache de la pure spatialité et de la 
coprésence temporelle » (ibid.) serait seule insuffisante pour enclencher cette vision de mondes multiples et de temporalités conflictuelles. L'exemple de la mort rend visible ce jeu entre les divers et parfois contradictoires mondes. Les Korowai «habitent leur présence humaine comme s’ils étaient remplis d’autres temporalités» (ibid.). Autrement dit, la présence n’est pas réductible au temps présent, dès l'instant où elle est toujours saturée par des connexions avec d’autres temporalités absentes, comme celle de la mort. Et réciproquement, lorsque les temporalités des hommes séparés par la mort sont elles-mêmes surchargées de la seule temporalité présente (ibid.). La mort est bien une «présence» sans laquelle la présence spatio-temporelle de la réalité ne pourrait pas advenir ni à la sensation ni à la représentation. La présence de la mort n'est pas la réduction symbolique de la présence sensible réelle. Les deux mondes cohabitent à égalité afin d'interagir dans un même univers métaphysique et cosmogonique, sans que l'un soit soumis à l'autre.

Ce que l'on constate dans le fonctionnement du présent et de la présence chez les Korowai, est en droit d'être étendu à tout le dispositif rituel en général et dans toutes les sociétés.

En témoigne par exemple le jeu auquel se livre la dualité présence/absence chez les Runa d'Amazonie occidentale. En s'appuyant sur les analyses de Peirce concernant la vie des signes, Eduardo Kohn (2013, p. 30) observe que chez les Runa, tous les signes existent toujours à propos d'un monde qui n'est pas toujours présent. Le signe - cri, son, bruit, nom, onomatopée -, lui, est bien présent dans sa contingence immédiate et compacte, signe sans lequel le monde auquel il est censé renvoyer n'existerait même pas. Le son émis par le singe ou le chien avertit de la présence feulante du jaguar. Le jaguar sera bien présent, mais pas immédiatement. Pour l'instant c'est le futur, et peut-être le futur de la mort, qui annonce et alimente la présence des signes, icônes ou indices.

Et il ne s'agit en rien de construire un monde symbolique, venant doubler le premier par une extension sémantique. Le signe, par son immanence, décrit bien ce monde-ci, hic et nunc, cependant il en trace des contours de simple écho, d'une présence compacte et auto-suffisante. Le Runa pourrait en rester là, mais il mettrait alors sa vie et la vie des autres villageois en danger. Nous ne sommes 
pas tenus ici par une logique de l'interprétation par laquelle le Runa serait libre de prêter telle ou telle signification aux signes captés. Nous aurions plutôt affaire à une logique iconique par laquelle le cri ou le bruit de l'oiseau ou de la feuille appelle inexorablement le danger, la mort ou la violence.

Comme l'écrit Kohn, le futur, pour le moment absent, «vient affecter le présent» (ibid., p. 37) ${ }^{3}$. Le Runa prend le signe présent comme anticipant la présence future, qui, pour l'instant, se vit sur un mode absent. Jusqu'au jaguar qui anticipe la mort qu'il va administrer à l'agouti. En guettant sa proie, le jaguar se «re-présente», par le truchement d’un certain nombre de signes présents (l'odeur, les bruits de succion, les sons des pas sur l'humus, les traces de bave d'un premier passage), l'agouti auquel il va s'attaquer dans un futur plus ou moins proche. Et pourtant le jaguar ne symbolise pas (ibid., p. 194-195). C'est pourquoi les Runa ne sont pas tant affectés par la présence charnelle des autres êtres de la forêt, que par leur «absence constitutive» de leur existence ici-bas (ibid., p. 2 Io). Au fond, ce que cherche à nous dire Kohn de la vie sociale et culturelle des Runa, repose sur une conception de la «la réalité comme étant plus que ce qui existe» (ibid., p. 216). Le monde des Runa est toujours un monde possible de relations à être.

L'action rituelle, comme la transe, la chasse, la prise de drogue, la cérémonie d'intronisation, implique toujours un jeu d'apparition/disparition de la présence et de l'absence du temps et de l'espace. Dans le même temps où le rituel fait appel à la présence hic et nunc de la chose visible sur laquelle le sorcier, le prêtre ou le chamane travaille, un autre monde est convoqué, non pas au titre de doublure symbolique du premier, mais plutôt comme son double. Il s'agit d'une absence présente et d'une présence absente. Le microcosme de l'action qui se déroule ici au présent ne peut avoir d'efficacité que s'il est accompagné d'un autre espace/temps plus large, macrocosmique, invisible mais rendu intelligible par la scène rituelle présente. Tout se passe comme si le monde microcosmique de l'action rituelle devait, pour faire sens et entraîner des réactions, s'étendre à d’autres mondes que seul ce premier éclaire et illumine. Il en va de même pour la réciproque. Le monde des morts et des esprits ne peut 
s'activer que sur le fond de l'action rituelle présente dans un monde d'ici-bas. La vérification s'opère à double sens (Stasch, 20I I, p. I60). Dans la mesure où une action rituelle sert à rendre vivante une croyance, ou une représentation cosmogonique, il est nécessaire d'utiliser des outils de visibilité pour ce faire, comme la danse, le port de masques, la transe ou le sacrifice. À l'inverse, pour croire dans l'action visible, il convient de l'investir d'une force ou d'une puissance qui n'est pas présente au moment de son effectuation.

Le rituel ne peut se concevoir, anthropologiquement parlant, que sur le mode de l'affectivité sensible. L’action doit bouleverser, remuer les sens, réorienter les perspectives de notre monde. Le monde korowai comme le monde Runa, aussi bien que toutes les pratiques rituelles, nous enseignent que la présence du rapport au monde est cela par quoi l'humain construit du sens par la voie et la voix - souvenons-nous du chien et de la vipère de mort - de la sensibilité.

\section{LA PROXIMITÉ DU LOINTAIN}

Cependant toute présence n'offre pas cette transparence à elle-même et cette plénitude qu'on serait en droit d’attendre de la position physique d’un être-là. Ce n'est pas parce que la chose ou l'objet se tient devant moi, bien visible, dans son identité apparente, qu'elle n'est pas travaillée par des fractures invisibles et des divisions déformantes. Être là ne suffit pas pour être compris, intelligible ou posséder un sens incontestable. L’ambiguïté accompagne aussi le visible et le sensible.

Par quel miracle, demande l'anthropologie, parvenons-nous, en dépit de différences abyssales et d'un éloignement dû à l'altérité extrême, à nous comprendre entre hommes de cultures diverses et multiples?

Pour Husserl, par exemple, il convient de ne pas confondre deux modalités d’approche du problème. D’une part, ce que je sais vrai de moi, il y a de fortes chances que cela le soit aussi de ceux «présents dansmon environnement» (Husserl, 1950 [1913], p. 93). À condition, précise Husserl, qu'il s'agisse du «même monde ». D’autre part, ce même monde peut être accessible à partir de points de vue différents, ce qui est susceptible de conférer à ce même monde des aspects qui le modifient. Ce sont les modes d'appréhen- 
sion (Auffassungsweisen, ibid.) qui différent, et non le monde dans son «objectivité » (Objektivität). Néanmoins, «en dépit de tout cela nous arrivons à nous comprendre avec nos voisins ». Le terme de «voisinage» peut être entendu de deux manières : soit la proxémie spatiale, soit la présence étrangère mais lointaine dans l'altérité culturelle. Et cette réalité «objective», Husserl la nomme «d’ordre spatio-temporel». En d’autres termes, il vise par là une présence qui n'est pas que sensible, mais qui relève aussi d'un être-là culturel.

On est en droit de réitérer, devant une telle analyse, le reproche dont Husserl fut tant accablé, à savoir celui de croire en un idéalisme universel. Car la présence dont il s'agit, en l'occurrence celle du voisin à tous les sens du terme, est loin d'être aussi pleine et signifiante que Husserl veut bien le dire. Husserl semble toujours parler d'une présence comme s'il s'agissait d'une expérience consciente d'elle-même, s'enveloppant comme vie, et tenant tous les bouts de l'expérience au sens d'«Erlebnis». Tout se passe comme si cette présence dont nous parle Husserl devait se tenir dans un fond originaire, toujours bouillonnant de vie (Henry, 2003, p. 44). Cette vie, qui donne la présence au présent, étant première, elle indique l'origine d'où surgirait l'être-là, celui du voisin comme le mien. Encore faut-il croire en cette «Ur-impression », que Husserl développe surtout dans les Leçons pour une phénoménologie de la conscience intime du temps (1996 [1928]). Sans cette condition première, déclenchant tout le reste, pas de présence.

L’anthropologie, en dépit de la finesse analytique de la réflexion husserlienne et de ses intuitions en matière ethnologique, ne peut se contenter de la stratégie du coup de force d'une croyance en un premier moment fondateur d'un monde, stratégie qui semble nous rapprocher du dispositif de la Révélation.

À l'opposé de l'analyse husserlienne, Jean-Paul Sartre nous offre une puissante démonstration de la présence, véritable épistémologie pratique, dont l’anthropologie pourrait tirer de nombreux bénéfices.

Dans L'Être et le Néant, deuxième partie «L'être-pour-soi», le premier paragraphe du premier chapitre s'intitule «La présence à soi» (Sartre, 1943). Sartre défend la thèse d'une présence qui ne parviendrait jamais à coïncider avec soi-même comme sujet. La présence à soi est cette distance de soi à soi qui n’aboutit jamais à faire 
la synthèse du sujet présent, mais dont on ne peut, malgré tout, jamais se passer pour se constituer un monde présent. En effet, «toute "présence à” implique une dualité, donc séparation au moins virtuelle. La présence de l'être à soi implique un décollement de l'être par rapport à soi» (ibid., p. I19).

Il n'y aurait ainsi de présence que trouée par une négativité qui viendrait en quelque sorte la subsumer et la fragiliser. Et c'est surtout dans ses relations avec le monde extérieur que le sujet expérimente cette douloureuse présence qui s'efface au fur et à mesure qu'il prétend s'en saisir. Toute présence, supposant une séparation préalable, est seulement en mesure de rapiécer ce qui fut décousu, dans un deuxième moment, dans un après-coup du temps. Ce qui sépare est non seulement ce qui fut, mais surtout le désaccord, le différend et le malentendu, bref tout ce qui rend inintelligible le frottement entre deux cultures. Et, c'est aux yeux de Sartre, cette fissure qui compose la présence de soi à soi et des autres à moi-même. Il doit y avoir «néantisation de l'identique» (ibid., p. I20) pour que, ontologiquement et ontiquement, cette présence apparaisse et fonctionne. Nous verrons, un peu plus loin, la proximité avec les analyses ethno-anthropologiques de De Martino.

Pour le dire en termes existentialistes, toute présence serait alors une aliénation dont on ne pourrait se passer afin de se constituer en monde humain. Et, citant Mikel Dufrenne, Michel Henry épouse intimement la démarche sartrienne, et nous fait apercevoir les conditions d’apparition de tout donné : «la possibilité pour qu’un donné apparaisse comme donné, c'est cette distance infranchissable et perpétuellement sauve d'où peut être discernée une présence » (Henry, 1963, p. 87 ; Dufrenne, 1949, p. 16).

La présence est bien ce qui tente d’unir ce qui fut désuni mais qui garde la trace de cette désunion. Dans le même temps où nous nous unissons aux choses présentes, la trace de l'impossibilité de l'attachement se manifeste, car c'est bien «la différence qui est l'essence de l'unité» (ibid., p. 89). Pour qu'il y ait présence, il est nécessaire que je diffère des autres et qu'ils diffèrent de moi. Ou, pour le dire autrement, c'est par la distance que la présence se construira. «C’est une proximité dans le lointain» (ibid.), écrit Henry dans une contiguïté affective à Hölderlin. Comme De Martino nous l’a montré dans ses études sur ses différents terrains italiens, «la présence s'obtient sur le fond du déchirement et de la division» (ibid.). 
Pour De Martino, si les humains, partout dans le monde, ont recours aux pratiques magiques, sorcellaires, exorcistes, occultes, maléfiques ou encore aux rituels de possession, c'est essentiellement en raison de la «fragilité de la présence» (De Martino, 1999 [1959], p. I08). Il s’agit, pour l'auteur du Monde magique, d'entendre par «présence» celle, personnelle, de l'expérience existentielle, soumise en permanence aux aléas des «crises de misère psychologique » (ibid.). Bien que son enquête concerne les villages de Lucanie en Italie du Sud, on est fondé à universaliser son propos et à en inférer une théorie anthropologique de la présence. Si la pratique magique consiste à protéger, c'est bien que la présence existentielle en éprouve un besoin vital. Cette présence personnelle ne se contente pas de se tenir quelque part, dans un espace-temps, en tant qu'être-là. «En tant qu'énergie opérante», elle incarne «le centre même de la positivité culturelle» (ibid.) Cependant, cette positivité offre le paradoxe d'être traversée par «la puissance du négatif». Que veut dire De Martino par «négatif» ici? Il faut l'entendre non seulement en son sens empirique ou ontique d'être miné par la «faim» ou la «maladie», mais surtout en son sens ontologique de «risques de naufrage de la présence individuelle». Le naufrage existentiel induit la perte d'identité et le déséquilibre de l'insaisissabilité de soi. La culture, en ce sens, n'est que la dynamique de réintégration des risques de crises et des crises elles-mêmes. C'est la raison pour laquelle elle porte en soi le fruit de sa désintégration, sous la forme de ce que De Martino appelle le «négatif». Mais, à un autre niveau, plus ontologique ou «métahistorique », la présence est négative car elle ne parvient pas à elle seule, à éviter la répétition de ces crises. Il faut donc avoir recours à l'horizon stable de «configurations symboliques » (ibid., p. Iı) qui consistent à «absorber» le négatif de la présence temporelle et spatiale. La présence individuelle, soumise aux accidents de l'«historicité du devenir», est alors en mesure de s'élever au niveau d'un «plan métahistorique», celui d'un horizon sémantique, que l'on appelle «culture» et qui provient, de ce fait, du travail térébrant du négatif (ibid., p. i i I). Cette analyse de la culture au travers de la présence individuelle s'applique aussi aux expériences de «vide» et de «dépersonnalisation » que De Martino extrait de l'analyse qu’en fait Janet en matière de psychiatrie. Et comme dans les expériences culturelles la présence 
individuelle n'est jamais seule, on est en droit d'inférer que les crises du vide et de la dépersonnalisation sont enfantées par la présence de l'altérité, celle de celui qui, en face ou à côté de moi, subit la même crise de la présence (ibid., p. Iı6).

Peut-être est-ce l'occasion ici d'opérer une mise au point d'ordre méthodologique et épistémologique. Ce que décrit De Martino, à partir de ses expériences de terrain en Lucanie, n'est rien d'autre que le monde de l'enquête de terrain ethnologique et, dans un second temps, celui de l'appréhension de l'humain en tant que tel, quelles que soient les cultures étudiées. L'altérité ne se positionne pas seulement comme un autre moi ou un autre que moi, mais aussi et surtout comme celui que je ne comprends pas. Aussi faut-il avoir recours à l'expérience existentielle de la crise afin de tenter de saisir en quoi les autres sont à même, comme moi, de sentir et de subir les crises de cette présence fragile qui conditionne l'humain pris universellement, à travers la souffrance, la peine, la maladie, la peur de la mort.

C'est donc par la sensibilité de la chair et de l'affect, comme nous l'examinerons plus loin, que l'universalité s'installe et légitime la possibilité d'une science anthropologique.

Dans Le Monde magique, De Martino affine son analyse de la présence en la divisant en deux moments. La première, qu'il exemplifie par la coprophagie du schizophrène, «déchoit du plan culturel qui lui reviendrait de droit», alors que la seconde, qu’il exemplifie par «la coprophagie magique», «exprime au contraire une présence non encore décidée, qui se fonde dramatiquement, et qui participe d'un monde culturel, d'un drame historique à caractère public, doué d'une valeur permanente de progrès dans l'histoire de la civilisation» (ibid., p. 197).

Il y aurait donc deux présences en cela que celle attribuée à la maladie mentale réelle ne fait que subir une situation, sans l'avoir construite et en l'absence d'un objectif symbolique à même de la traduire en termes culturels, alors que la seconde dévoile une élaboration symbolique de traduction d'une situation dramatique qui vise à construire l'identité d'une culture, comme le rite ou la cérémonie du sacrifice dans de nombreuses religions. C'est la seconde qui intéresse l'anthropologie dans la confection de ses objets et de ses catégories, 
car elle pose le redoutable problème du sens que les sujets humains accordent aux valeurs et aux principes qui président à leur culture. Présence et culture sont, une fois de plus, intimement liées, puisque les manifestations culturelles doivent pouvoir être vues à travers une présence pour être crues. Dans un autre passage du Monde magique, en s'appuyant sur des exemples relevés sur différents terrains, De Martino illustre ce qu'il appelle «la volonté d'être là comme présence face au risque de ne pas y être» (ibid., p. 95). Aussi bien chez les Malais que chez les Toungouses, il existe un phénomène d'échokinésie qui consiste à imiter immédiatement les gestes de quelqu'un en les exagérant jusqu’à l’absurde ou à la limite d'un risque de mort feint. Pour De Martino, nous sommes face à une présence improductive car elle se contente de mimer sans visée active en subissant une première action (phénomènes latah ou amok pour les Malais et olon pour les Toungouses, ibid., p. 92-93). Dans ces deux exemples, «le contenu est perdu en tant que contenu d'une conscience présente», car seule la forme de l'imitation reste. Entre la présence et le monde que le sujet imite, il n'y a plus de différence car celui-ci «devient arbre» ou feuille en s'y confondant. Le sujet perd sa présence autonome et souveraine, puisque cette dernière «se comporte comme un écho du monde» (ibid., p. 94). En revanche, dans le magisme, la volonté de lutter contre l’adversité des risques appelle «la défense et le rachat: la personne cherche à intégrer sa présence échappée» (ibid., p. 95). La culture devient ainsi une lutte à mort afin de maintenir vivants les signes et les symboles de sa présence. Pour qu'il y ait culture et, partant, symbolisation, il est nécessaire qu'il y ait opposition, travail du négatif. Et la force de la magie, ainsi que de toutes les entreprises rituelles, consiste, en créant des institutions adéquates (le magicien, le chamane, les danses, les masques, les cérémonies d’intronisation, les sacrifices, etc.) à «signaler et à combattre le risque» (ibid., p. 21I) suprême : celui de disparaître dans le néant du non-sens ou de la mort.

Pour bien saisir l'enjeu de cette épistémologie de la présence, il conviendrait peut-être de s'intéresser à la manifestation et au fonctionnement de ce qu'il est convenu d'appeler un rituel. Certes, de nombreux travaux ont été consacrés à cette pratique dont la confusion et l'obscurité le disputent à une diversité telle qu'on abandonne trop souvent le souci d'en clarifier le sens. Que nous portions 
notre attention aux rituels chamaniques d'Amazonie et de Sibérie, ou à ceux d'Afrique de l'Ouest ou du désert australien central, des constantes ne manquent pas d'apparaître. Et, au-delà des différences et des contradictions, une ligne de force s'impose: un rituel rend présent ce qui, par définition, n’aurait pas pu se manifester autrement. Cette présence de et par l'action d'une manipulation, d'une danse ou d'un sacrifice, telle «une carte» donne à voir, en un tour de force de la métamorphose des corps, «dans un petit espace sensible une image d'ordonnancements spatio-temporels plus grands et plus diffus » (Stasch, 201 I, p. I62).

En tant quaction, le rituel n'étant finalement que cela, et non en tant que représentation symbolique, il se projette dans un monde absent, celui des dieux, des ancêtres, des esprits et des morts, et y précipite la réalité du monde de l'icibas, du «Tu es ici». Cependant, le rituel ne procède pas sur un mode restrictivement «iconique», ne se contentant pas de créer des images, mais montre l'autre monde en l'indexicalisant. Le rituel ne se satisfait pas de présentifier une absence, ce qui le conduirait à recharger à nouveau la théorie des deux mondes hiérarchiques par l'opération culturelle classique du travail symbolique. La danse chamanique ou la transe sont les deux mondes parallèles, sans primauté ni préséance de l'un sur l'autre. Ce dispositif est rendu possible par les procédés techniques liés au corps, mais aussi et surtout parce que c'est le corps, et lui seul, qui abrite et habite le monde de la douleur, de la souffrance, de la pulsion et de la chair. Le rituel n'est pas le symbole d'un autre monde, il est les deux mondes à la fois, celui des corps qui souffrent et celui des dieux qui les montent. Car c'est bien par le corps ancré dans l'espace et le temps, sans lesquels aucune présence ne pourrait advenir, que les expériences des deux mondes sont à même de se rejoindre. Un danseur ou un sorcier est bien le signe qui rend présent, en fabriquant en quelque sorte cette présence, un dieu ou un mythe, mais sans pour autant s'effacer lui-même en tant que présent. Il met en scène deux présences : la sienne et celle d'un autre qu'il indexicalise (ibid., p. i63).

Par voie de conséquence, dans le rituel, la distance entre le corps qui reçoit ou qui subit et la source de la souffrance ou du plaisir s'abolit. C'est le même corps qui, loin de symboliser une altérité productive et extérieure, l'actualise au contraire en la rendant présente devant les yeux. Les partici- 
pants ont l'habitude de dire: je vois les dieux, je vois bien les morts tout en voyant celui qui les incarne ou les porte.

Michael Jackson (1998, p. 132) fait justement remarquer que les Warlpiri du territoire septentrional australien se réfèrent à deux présents, le premier qu'ils appellent «palka» et qui est censé désigner le présent incorporé dans un corps, le deuxième qu'ils appellent «lawa» et qui est censé indiquer une absence présente. La relation entre les deux peut être assimilée à une «oscillation » perpétuelle comme celle qui rythme le jour et la nuit, couple inséparable dont les membres s'appellent irrémédiablement, ou encore celle qui scande le réveil et l'endormissement. La logique qui préside aux relations entre les deux présences est celle de la réciprocité inéluctable.

Où l'on retrouve la logique de la «parité » non identique chez les Korowai qui montre que les paires, par lesquelles ils indiquent et définissent les choses, les objets et les personnes, n’enveloppent pas «une entité telle une présence en soi, mais une présence définie par ses liens avec quelque chose qui est absent » (Stasch, 2009, p. I00).

Ce qui revient à admettre qu' «une personne est l'autre dont la personne provient» (ibid., p. I04.). Elle est donc bien deux sous la forme d'une même présence. Et il en va de même pour le présent, que le temps permet de ne pas être contenu dans un en-soi souverain.

Les expériences de la ritualité révèlent ainsi aussi bien chez les Korowai qu'en Ligurie, dans le Nord australien ou en Amazonie, que les objets, les choses et les personnes, dans le même temps où leur présence s'avère incontestable, y échappent en quelque sorte par une espèce d'incomplétude qui en marque à la fois l'absence et la parité avec quelque chose d'autre dont elles ne peuvent pas se défaire. La présence ne serait alors présence que par cet écart infranchissable qu'elle entaille par rapport à elle-même. En même temps que la personne occupe une place présente à elle-même incontestable qui se manifeste par le cri de la souffrance ou du plaisir et qui semble nous dire «ici je me tiens» (Ricœur, 20I3, p. 4I2), «l'être humain n’en a pas la maîtrise ». Cette ontologie de la présence se vide de l'existence même dont elle procède. Tout se passe 
comme si le jeu de l'être-au-monde dans toutes les cultures devait simultanément s'enraciner dans une présence corporelle marquée par la finitude de l'espace et du temps, et par l'appel à une extranéité qui devait en sceller l'inhérence.

Pour être de ce monde, le chamane, le sacrifiant ou le magicien n'en indexicalisent pas moins un autre monde absent qui autorise le premier à être présent.

\section{DE LA SENSIBILITÉ}

Si quelque chose devait exhiber la présence, comme un fait en train de s'accomplir, comme une évidence irréfragable et comme l'immédiateté la plus proche possible de soi, ce serait sans nul doute celle du corps, compris comme chair et auto-affection.

Si je souffre, si j’ai mal, qui pourrait prétendre le contraire? Ma douleur et ma souffrance sont ma propre preuve et son témoignage à la fois. Je suis ma propre douleur. Et c'est le corps, en tout premier lieu, qui, par l'intimité qu'il établit avec la vie immédiate, inscrit la sensibilité comme au fer rouge. Le corps, comme Husserl et Merleau-Ponty l'ont bien montré, est «chose sentante», et sentant-sensible (Husserl, 1982 [1952], §I8c). D’où pour Husserl l'usage de Leib en allemand, à savoir, à l'encontre de Körper, le corps propre «celui qu'on sent du dedans» (ibid., p. 408). Mais fait remarquer le traducteur, on pourrait tout aussi bien traduire Leib par chair, en mettant ainsi l'accent sur son «aspect-sensible» et non pas sur «l'aspect-stature».

Nous n'avons plus affaire à un corps fragmentable, mais à une totalité indéfaisable, en cela que la douleur est mienne, mappartient en son entièreté et envahit l'intégralité du corps. Mieux, car sans le corps propre, l'orientation dans l'espace ne pourrait s'opérer. Il m’oriente et structure ainsi mon monde spatial (ibid., p. 93).

Et il en va de même pour le corps qui expérimente les choses sensibles du monde extérieur. Non seulement, je touche la table, y reconnaissant sa rugosité ou son lissé, mais je suis en mesure de me toucher touchant la table. Dans l’acte sensible, nous sommes donc en présence de trois événements : le monde extérieur, ma main le touchant et ma deuxième main touchant la première. 
À quoi une telle analyse peut-elle être utile à une réappropriation de l'anthropologie sur des bases renouvelées?

Husserl, dans le même texte, s'emploie à fonder la collectivité des personnes, quelles qu'elles soient. Et il amorce son analyse en défendant la thèse de l'appréhension des autres par l'«intuition» (ibid., p. 322). Or, il convient d'éviter d'entendre par là une saisie vulgaire et primaire du monde extérieur, y compris des autres. Ce que Husserl veut dire par là c'est que «nous trouvons [toujours] des hommes d'un seul tenant qui ont commerce avec nous, et les corps font partie intégrante de l'unité humaine» (ibid.) Ce que Husserl appelle la «teneur intuitive » est bien la «corporéité de chair » : le geste, le mot, le jeu de physionomie, l'intonation, bref ce par quoi les autres personnes me sont données dans l'expérience spatio-temporelle de toute enquête. Mais l'expérience de la découverte des autres ne se limite pas à la corporéité, elle s'élance vers «la vie spirituelle des personnes, leur pensée, leur sentiment, leur désir, leur façon de s'affairer» (ibid., p. 323). L'intuition veut dire ici la saisie immédiate par le corps propre. Et dans l'acte intuitif par exemple qu'est «la mimique», je rattache un sens «au-dedans de la conscience de l'autre ». Comment cela peut-il se passer? C'est en raison, nous explique Husserl, de la relation causale que, dans ce type d'expérience anthropologique, je suis contraint de congédier. Pour saisir les autres personnes de l'humanité, la relation étiologique ne m’est d'aucun secours. Car entre la chose «tête » et le visage de l'autre d'une part, et le visage tel qu'il m’apparaît d'autre part, il n'y aucun rapport de cause à effet (ibid.). Il y aurait un saut qualitatif que, de toutes les façons, je ne comprendrais pas. La société, pour Husserl, est le lieu dans lequel, «la représentation compréhensive que les autres ont, ou encore peuvent avoir de moi, me sert à mappréhender moi-même en tant qu'homme social» (ibid., p. 332).

Et c'est seulement ainsi que je suis en mesure de me ranger « dans la collectivité humaine» et seulement ainsi aussi que «je peux dire «nous»». Et dans tout ce dispositif, nul besoin d'un «processus de “naturalisation”» (ibid.). 
L'analyse de Husserl peut rendre un service capital à l'anthropologie dans la mesure où elle met en évidence la relation charnelle au sein de la relation socio-culturelle. Il ne peut y avoir de corps senti qui ne présuppose un autre corps sentant. Et nous pouvons le vérifier dans de très nombreuses sociétés où la relation entre les humains est pensée et vécue comme un lien sensible indéfectible, à partir du moment où ils appartiennent à la même tribu ou au même clan. Les corps forment ainsi un seul et même corps sans discontinuité, comme chez les Korowaï de Papouasie occidentale ou chez les Canaques de Nouvelle Calédonie. Il s'agit bien en effet d’une présupposition, c'est-à-dire d'une condition de possibilité afin que le monde humain, mais aussi animalier, soit concevable. Les corps ne viennent pas se surajouter les uns aux autres pour former société, encore moins se juxtaposer. Ils présupposent les uns les autres que la société est possible et réelle. Or les corps se manifestent dans la présence de leur «prestation» (Henry, 2000, p. 158). On n’échappe pas à sa souffrance signifie que la souffrance est auto-pathétique et que cette auto-affection me lie indéfectiblement aux autres à égalité. Ce sont les expériences de l'affect qui l'emportent, dans de nombreuses sociétés, sur les lois de la représentation ou de l'intentionnalité : au cours de la chasse, de la pêche, des cérémonies rituelles, des prières, des sacrifices. Le message husserlien est celui qui pose la communauté humaine comme relevant avant tout d'une incarnation «pulsionnelle qui se situe sur le plan du pathos » (Henry, 20IO, p. 4I). Nous sommes en droit de poser ainsi les fondations d'une anthropologie matérielle et réaliste: nous devenons humains en nous saisissant des corps qui souffrent ou qui jouissent.

C'est par le corps-propre, par la chair, que la présence au monde et du monde s'opère, y compris celle des autres. Et comme Marcel Mauss nous l'a magistralement montré, l'univers symbolique lui-même s'origine et s'incarne dans le corps. La posture des corps met en présence et rend présents «les symboles moraux ou intellectuels » (Mauss, 1968 [1934], p. 372).

L'incarnation dont il s'agit chez Husserl, Henry ou Mauss ne se réduit pas à des représentations mentales du corps ou encore à son objectivation naturelle ou scientifique, qu'elle soit physique ou biologique (Csordas, 1994, p.xi). Dans la douleur, par exemple, comme l’anthropologie médicale ou religieuse 
nous le montre, le corps n'est pas un vecteur ou une médiation qui nous conduirait d'un monde sain vers un monde malsain ou malheureux. Il serait plutôt le pivot central à partir duquel le monde se donne à moi dans toute son indétermination. La douleur me rend le monde douloureux dans toutes ses occurrences. Ce fut le message ultime de Mauss : le corps est certes assimilable à un outil puisqu'il me permet d'opérer sur le monde par la main, le doigt, le bras ou le pied. Cependant, au-delà de cette fonction d'outil, bien connue depuis Aristote, il convient de l'approcher comme la source d'une appréhension holiste dans la mesure où c'est toute la culture qui prend sa racine en lui. Le meilleur exemple reste encore celui de l'émotion qui, en s'exprimant, incarne les humains dans un rapport socialisé. Dans les études d'anthropologie religieuse, les esprits, lorsqu'ils s'emparent des orants ou des sacrificateurs ou des chamanes, se saisissent avant tout des corps afin que la marque de l'émotion apparaisse aux autres intervenants. Pas de possession sans incarnation, donc sans chair. Le mal ou le démon ou le diable doit bien être ressenti à l'intérieur de son corps dont la douleur sera le marqueur indélébile. Si lors d'une cérémonie de possession ou de transe, le corps ne se manifeste pas par la présence de ses gémissements, de ses vents, de ses cris, de ses halètements, de son souffle, de son tremblement, de ses rires et de ses pleurs, aucune expulsion du mal ou présence du sacré ne pourra se réaliser. La culture s'enracine dans le partage communautaire des sensations les plus douloureuses entre les acteurs et le public. Sans cette expérience commune, aucune culture collective ne peut venir au jour. Dans de nombreuses sociétés, comme par exemple chez les Runa d'Amazonie occidentale, le langage est littéralement vécu comme l'expression sensible du corps, à savoir d’abord et avant tout comme une litanie de bruits. C'est ainsi que la pensée s'origine, dans la douleur d'une profération et l'invective d'une adresse. «Il n'est aucune sorte de sensation qui soit plus vive que celles de la douleur; ses impressions sont sûres, elles ne trompent point » (Sade, cité dans Perret, 20I3, p. I24).

Peut-être touchons-nous là la pointe extrême sur laquelle viennent se rejoindre la nature et la culture. 


\section{L'ESPACE EST LE TEMPS}

Dans toutes les sociétés et les cultures du monde, les identités se construisent à travers l'espace et le temps, certaines d'entre elles insistant plus sur le premier paramètre, d’autres sur le second. La civilisation occidentale, sans doute en raison de son enracinement chrétien, a privilégié le temps comme facteur constitutif de l'identité individuelle et collective, afin de bien distinguer la temporalité humaine finie et l'intemporalité divine infinie. Il est intéressant de comparer un tel schéma avec les modalités existentielles d'autres cultures. Par exemple, en Amazonie, les rituels fort nombreux et extrêmement différenciés, ont pour fonction de présenter, c'est-à-dire de rendre présents des ordres, des normes et des comportements dans un espace réduit et commun. Par un processus iconographique et indexical la société s'approprie l'occupation spatiotemporelle de chacun. Tout est ramassé dans le rituel qui fait voir, en miniature et en raccourci, les méandres et l'entrelacement des divers ordres parmi lesquels se meuvent l'individu et la collectivité. Au lieu de découper l'existence en tranches successives et chronologiques, le rituel amazonien groupe et agrège, par scénographie interposée, les activités auxquelles doivent se livrer les agents pour accomplir leur être-au-monde. C'est la raison pour laquelle, il peut nous sembler incompréhensible d'assister à une concentration de la vie quotidienne et de la ritualisation permanente de l'existence sociale. Ce que Stasch (201 I, p. I62) appelle «une densité poétique de signification» essaie de traduire cette situation existentielle des places occupées par les agents dans un espace ritualisé. Graham (1993), de son côté, montre par l'étude de «l'éloquence masculine dans une société amazonienne, comment l'organisation spatiale et sensorielle des corps qui y participent, à travers leurs voix physiques, contribue à la qualité de l’administration politique» de la société (Stasch, 20II, p. I62).

Et pour revenir aux Korowai, la distance entre les habitations et leur dispersion constitue un moyen pour «maintenir l'autonomie et l'égalité» (Stasch, 2009, p. 4). Autrement dit, se séparer spatialement traduit le désir d’appartenir au même monde. Et l'altérité spatiale est pensée par eux comme le meilleur moyen de se rapprocher les uns des autres. Une trop grande proxémie spatiale signifierait qu'il leur serait impossible de voir, toucher et sentir la forme géographique de la relation. L'espace 
est donc bien vécu comme devant marquer un intervalle pour mieux mesurer la bonne distance, dialectique entre le lointain et le proche, afin de cohabiter et de coexister. La meilleure intimité serait, selon les Korowai, celle qui permet de tenir loin le voisin, mais dans une proximité qui le rende présent (ibid., p. 259).

Rappelons ce qu'il conviendrait d'entendre par «spatialiser». Il s’agit de délimiter et de distinguer plusieurs espaces afin d'en mesurer tantôt ce qui les différencie, tantôt ce qui les rapproche, et, souvent, les deux à la fois. L'espace, à l'instar de la frontière, sépare et joint. Cependant, par l'écart, l'individu institue deux actes apparemment contradictoires dans la mesure où il rompt et soude simultanément. C'est exactement ce à quoi s'emploient les Korowai, dans le but essentiel à leur survie, d'éviter la guerre. C'est bien la notion d'étranger intime, d'externe interne, qui trouve ici ses limites et sa résolution. En spatialisant, je qualifie une identité par le jeu incessant du rapprochement et de l'éloignement. Je perçois ainsi autrui par la petite focale du microscope et par la grande focale de la lunette astronomique. L'espace, pensé comme un aller-retour permanent, permet ainsi de gérer dans toutes ses dimensions la présence des autres qui, sinon, pourrait être ressentie soit comme un envahissement, soit comme un effacement dans l'horizon d'un trop grand lointain.

Néanmoins l'espace ne peut se réduire à un simple acte de présence. Il doit aussi indiquer ce en quoi il matérialise le présent. Qu'est-ce en effet qu'être-là ou mieux, qu'est-ce qu'habiter un lieu? C'est «se trouver là», pour reprendre la belle formule de Wittgenstein, reprise par Heidegger. La contingence du «Befindlichkeit» (Ricœur, 20I3, p.407) est bien rendue par la double expérience du pâtir et de l'agir. Je pâtis en me soumettant à un espace contraint qui me possède, mais, simultanément, j’agis cet espace, par le ressouvenir de mes ancêtres ou par les activités de la chasse, de la pêche, de l'élevage, de l'agriculture, etc. Comme disent les Korowai «se trouver là» est à la fois étrange et intime. Dans tous les cas, loin d'être réduit à une simple res extensa, le corps se mue et se meut en chiasme charnel, grâce à la relation réglée aux autres. Sans être capable d'être situé et de se situer, le sujet humain se trouverait dans une impasse anthropologique, sauf à s'extraire d'une conception statique et inactive de l'espace. En étant coextensif à l'espace, le Korowai s'y inclut, ou plutôt, y est inhérent, mais pour aussitôt s'en détacher afin de pouvoir y revenir pour effectuer des actes qu'il ne pourrait autrement identifier. 
Dans les expériences existentielles de ritualisation, sacrifice, divination, transe, les acteurs recherchent la meilleure manière de suspendre le temps en créant un présent éternel. À cette fin, chaque geste doit montrer qu'il entame un nouveau commencement, de telle sorte que la répétition donne ce sentiment d’un présent à la fois étalé et suspendu. Et ce dispositif est permis par l'incarnation sensible des douleurs, joies, souffrances, jouissances liées aux divinités incorporées. La présence du sacré doit se voir dans un présent dilué dans son rapport à l'autre monde.

On pourrait paraphraser Husserl en affirmant que le temps est un vécu qui dure, et «avec cette durée il s'ordonne à un continuum sans fin de durées» (Husserl, I950 [1913], p. 275). C’est bien que le présent, en s'élargissant, a pris le pas sur les autres modalités du temps. Et cet élargissement est autorisé par la présence vécue de l'espace dans lequel et par lequel évolue le corps souffrant ou jouissant, bref par la chair. Et si le présent semble avoir tout envahi par l'effet de son étalement et son étirement, c'est parce que, comme le suggérait Saint-Augustin, il y a un présent pour chaque mode de la temporalité : «Le présent du passé, c'est la mémoire; le présent du présent, c'est l'intuition directe (contuitus, attentio); le présent de l'avenir, c'est l'attente» (Saint-Augustin, 1964, p. 269).

\section{AU-DELÀ DU SYMBOLIQUE}

La quotidienneté et l'ordinaire de l'existence, la spatialisation de la chair dans le voisinage et dans les foules, la facticité des corps et des actions en l'absence de tout cadre symbolique en arrière-plan nous contraignent à assumer une discontinuité phénoménologique par laquelle les choses et les images des choses sont ce qu'elles sont, dans la présence de leur ontologie. Les symboles sont toujours affectés d'un sens caché à explorer, les choses, quant à elles, étant des symptômes non nécessairement référentiels et visibles dans leur manifestation même. Dans les poèmes de Francis Ponge, une chose, hors de toute destination symbolique, s'impose par une présence qui déchire le déroulement continu du présent existentiel. Le savon, par sa matière, ses lignes, sa couleur, pénètre par effraction, creusant une discontinuité, dans la continuité du temps qui s'écoule. La présence domine par sa fragilité même 
puisque le morceau de savon peut disparaître, se diluer ou s'évaporer. Les choses de Ponge seraient ce qu'elles rendent visible: un monde non donné dans sa continuité, mais seulement dans sa fragmentation et sa contiguïté. C'est sans doute ce à quoi songeait Henry lorsqu'il parlait de la manifestation d'une présence. Ce à quoi nous invitent Stasch, De Martino ou Kohn dans le registre ethnologique, Henry et Husserl dans un registre plus philosophique, c'est à examiner sur nouveaux frais la structure fondamentale de l'expérience existentielle elle-même et non plus comme signe ou symbole de quelque chose d’autre qui serait désespérément absent.

La présence confère un sens intensionnel sans avoir recours à une référence extensionnelle. C'est pourquoi l'essence même de la présence et de la manifestation des corps, des choses, des être-là, c'est l'aliénation, c'est-à-dire l'endossement d'une séparation sans laquelle aucune proximité ne pourrait avoir lieu. La leçon anthropologique des Korowaï, des Runa, des Italiens méridionaux et de tant d’autres sociétés et cultures se résume à cette présence qui ne peut s'obtenir que sur fond de déchirement et de division. Tout se passe comme si ces collectifs se tenaient au plus près d'une attitude naturelle d'avant la donation du sens, du symbole et de la représentation. C'est dans cette mesure que la sensibilité peut constituer une boussole dans notre rapport au monde qui est donné, primordialement, comme ce qui touche et émeut.

Nous avions déjà fait observer que le symbole est un instrument à double tranchant (Affergan, 20I2, p. 225). Il représente et présente à la fois la chose à laquelle il renvoie. Par «présenter», il convient d'entendre le fait de mettre sous les yeux. Cependant, il s'agit bien d'une fausse présence, puisque, comme Cassirer le fait remarquer, il est pris comme «simulacre» (cité dans Affergan, ibid.), mais «auquel rien ne correspond immédiatement dans les données sensibles mêmes ». Ce double aspect du symbole, qui constitue toute sa richesse, met parallèlement en évidence sa faiblesse puisqu'il ne parvient jamais à se substituer réellement à la chose. Il n’est pas la présence, il y subroge seulement. D'une part, «ventriloque d'un monde absent», et d'autre part, s'il désigne bien la présence de la chose, c'est en tant que «chose en soi, close sur elle-même » (ibid., p. 226). Si le symbole indique bien la chose, c'est en tant que 
forme seulement, ou comme un schème. Tout le monde sait que le schème du chien n’est précisément pas le chien réel, matériel ou empirique, mais ce qui permet de le reconnaître en tant que chien. Il ne mord ni n'aboie. Autrement dit, le symbole «travaille toujours sur un vide référentiel» (ibid., p. 227).

Mais l'activité symbolique permet aussi de découper la réalité, par définition continue, en séries de catégories intelligibles pourvues de signification. Le symbole autorise donc le passage de la continuité spatio-temporelle ressentie comme telle, même si l'on sait aujourd'hui qu'il s'agit d'une illusion, à une logique discrète qui conditionne notre compréhension de la réalité sociale et culturelle grâce à l'activité de la fragmentation et de la sélection. En découpant, j'isole, je classe et je rends cohérente une réalité qui, sans cela, apparaîtrait confuse et obscure car subie dans un flux permanent où le sujet se contenterait d'une passivité cognitive. Cependant ce que l'activité symbolique rate, dans cette logique transformationnelle d'un espace continu à un espace de la signification, c'est l'entrelacement des corps et des regards qui occupent un espace pathique. Une ethnographie des corps propres, comme Stasch et Kohn nous la proposent pour l’Amazonie et la Papouasie Nouvelle Guinée, envelopperait ainsi la chose physique, la matière avec ses propriétés réales : le lisse, la coloration, le dur, la chaleur. Par ailleurs elle assumerait l'existence de la sensation pure : la chaleur du dos de la main, le froid aux pieds, le contact au bout des doigts, etc. Le sujet n'existe alors que s'il existe un monde où je me situe en tant que corps. La souffrance et la douleur sont à elles-mêmes leur propre fin.

Paul Ricœur trace une destination de l'activité symbolique fort éclairante, mais qui ne parvient pas à s’affranchir du lest représentationnel : «Le symbole n'est pas un retour aux signes naturels; il suppose un langage conventionnel qui a rompu avec la ressemblance sonore » (Ricœur, 20I3, p. I82).

La faiblesse ontologique et épistémologique du symbole est affirmée. À la différence de l'icône et de l'index, le langage symbolique est pétri de convention, en amont et en aval de la procédure de donation du monde. En l’absence de la chose, le symbole présentifie bien, mais toujours dans l'espace de la représentation. Jusqu'à l'action qui ne puisse être présente que par le truchement de 
ce qui la signifie en surplomb, en aplomb, du dehors, faute de quoi cette «action humaine ne serait ni articulée, ni médiatisée, ni interprétée» (ibid., p. 277).

Les Runa de l'Amazonie équatorienne n'ont pas une relation symbolique aux pumas, aux singes ou aux chiens, mais une relation indicielle, à savoir qui ne renvoie pas à une absence, mais convoque une présence charnelle, par le truchement du cri ou de la touffe de poils (Kohn, 20I3, p. 32). Ou plutôt, si absence il doit y avoir (puisque la bête n'est pas là devant les yeux), c'est d'une anticipation ou d'une prémonition qu'il sagit. Le cri entendu dans le lointain ou la trace de sperme ou de bave laissée sur la terre préfigurent l'approche de la bête qui sera présente dans un futur très proche. Car le signe est vivant. Un craquement de branche ou un souffle de vent dans les feuilles n'est pas une chose, mais l'élément d'une vie commune avec le sujet présent. Le monde des vivants est commun à tous (ibid., p. 33). «Nous vivons dans un monde qui excède le symbolique » (ibid., p. 38).

Et là où la logique symbolique est dépassée, c'est lorsque le symbolisant n’est pas distingué du symbolisé, mais fait corps avec lui. C’est cela que les Runa nous apprennent. La brindille cassée n'est pas le symbole de la bête qui vient de passer et qui a l'habitude de les briser en rampant. Elle est la bête en cela qu'il n'y aucun autre indice ou icône possible qui puisse appartenir à quelque chose d'autre qu'à cette bête. La brindille écornée est la bête, non pas en fonction d'une logique de la ressemblance ou de la similitude, mais en fonction d'une logique de l'identité commune partagée. Un iguane ou un puma brise les branches ou les brindilles lorsqu'il passe dessus car c'est son monde et c'est ainsi qu'il procède, jamais autrement. Il serait absurde pour les Runa de prétendre affirmer que la brindille brisée est le symbole de l'iguane ou bien le représente. Il en va de même pour le chien qui comprend, «sit» en langue runa, non pas au travers d'un système symbolique, mais parce que «sit» a une fonction indexicale (ibid., p. 53). Au fond, et en s'appuyant sur la réflexion de Peirce, Kohn défend l'idée d’icônes et d’indices, à la différence des symboles, «qui conserveraient leurs qualités intrinsèques indépendamment de leurs interprétants » (ibid., p. 207).

L'icône et l'indice s'emparent de l'absence comme si c'était une présence car le Runa entend et voit le jaguar devant lui, grâce aux bruits ou aux traces, comme si c’était le jaguar réel. 
Que certaines sociétés et certaines cultures déploient des activités pratiques et cognitives non symboliques ou asymboliques, s'affranchissant ainsi des contraintes liées à l'intentionnalité, à la promesse ou au projet, devrait alerter l'anthropologie sur le statut conféré au rôle de la raison et du langage dans leur dimension désignative, iconique et indexicale.

Par exemple, commander c'est agir ou faire agir. La fonction du commandement est d'emblée performative au sens illocutoire et non perlocutoire du terme. Tout l'édifice symbolique, représentationnel et objectiviste du langage et, partant, de la raison, s'effondre. Le commandement fait voir, montre, mais n'affirme rien. Inutile de faire remarquer que l'ordre symbolique ne se dissimulant plus derrière le monde à voir et à faire, et à l'encontre de ce que la vox populi de la science croit, le dispositif herméneutique et exégétique classique s'efface. La logique apophantique manquant, la machine à fabriquer du sens s'enraie et se réoriente vers de nouvelles logiques existentielles et sensorielles que nous avons observées chez les Korowaï, les Runa, les Sardes, les habitants de la Lucanie et chez les Samoans comme nous allons le voir tout de suite grâce aux analyses d’Alessandro Duranti.

Aux îles Samoa, Duranti (2015) a travaillé sur les relations entre les actes, les paroles et les intentions des individus. Il s'est vite rendu compte que, pour les Samoans, ce n'était pas tant les intentions des actions qui comptaient que le déroulement de celles-ci et leurs effets et conséquences induits au présent devant des témoins. (ibid. p. 63). La responsabilité de l’agent est donc liée aux effets et conséquences d'une action et non à ce qu'il comptait faire ou dire. C'est la raison pour laquelle le souhait, le vouloir dire, l'espoir ou l'attente de l'action sont moins pertinents et efficaces pour les Samoans que ce que cette action a réellement accompli, y compris sans que l'acteur l'ait voulu. L’action est donc réelle «lorsque les actes de la personne sont donnés en public et de telle sorte qu'ils puissent être évalués par les autres qui peuvent être affectés par les conséquences de tels actes » (ibid., p. 73) ${ }^{4}$. 
Seule la présence semble témoigner de la valeur de l'acte et non la conformité à une décision ou un commandement dont il faudrait attendre la réalisation pour juger de la légitimité de cet acte. Il est intéressant de noter, à ce sujet, qu’en langue samoane, le terme de «promesse » n'existe pas (ibid., p. 98), puisqu’une promesse est par définition trop éloignée de l’action pour pouvoir en évaluer la responsabilité et la légitimité. En soi une promesse n’est présente qu’à travers sa profération symbolique. Si intentions il doit y avoir, Duranti fait observer, comme beaucoup d'anthropologues, qu'elles devraient être prises en compte «dans le contexte culturel $[\ldots]$ non séparé des membres réels, en chair et en os, et qui possèdent leurs propres biographie, croyances et connaissance» (ibid., p. I55).

Le traitement de l'action chez les Samoans ressemble aux improvisations du jazz et aux activités spontanées qui caractérisent le flux de la vie quotidienne. Des événements imprévus parsèment la vie de tous les jours, lorsque, brutalement, je dois me diriger vers la gauche d'une route pour éviter la collision avec un obstacle imprévisible, ou lorsque je dois madapter soudain à une situation vis-à-vis de laquelle je n’ai pas le temps de réfléchir aux tenants et aboutissants de mon acte. Quant au jazz, c'est l'exemple prototypique où «la compréhension du processus créatif repère l'action en dehors de l'esprit de l'individu et la voit comme indépendante de l'intentionnalité ou de la volonté » (ibid., p. 225).

On est donc en droit de faire l'hypothèse qu'avec les Samoans, nous serions en présence d'une culture performative, mais autant au sens «perlocutoire du terme, prenant en charge les effets des actions, déclarations incluses, qu'illocutoire à l'instar d'un acte communicationnel » (ibid., p. 240). Husserl avait développé, comme le rappelle à juste titre Duranti (ibid., p. 29 et p. 238), une conception de l'intentionnalité beaucoup plus complexe que celle exposée trop souvent par le discours des sciences sociales. Pour Husserl, l'intentionnalité est constamment à l'œuvre au sein d'«un courant présent vivant» (ibid., p. 29) et non séparément ou a posteriori. L’intention ne vient pas se greffer sur la conscience qui se projette vers l'objet. Elle lui préexisterait plutôt en cela qu'elle se tient dans un espace pré-phénoménal, avant même la donation du sens. C’est cette opération que Husserl appelle «synthèse passive », 
dans et par laquelle un monde constitué d'objets primitifs et coextensifs existe avant que la conscience n'en élise un qui sera intentionnalisé.

Autrement dit une coprésence serait à l'œuvre avant même le remplissement de la visée d'objet particulier. Nous retrouvons ainsi l'exemple de l'improvisation de l'action comme lorsque «une piqûre d'insecte nous fait retirer la main involontairement» (Husserl, 1998 [1966], p. 165), la réalisation de la visée spatiale s'effectuant après coup. Tout se passe comme si l'intention chez Husserl ne recouvrait pas ce sens d'une visée particulière vers un seul objet animé par une conscience claire. Bien au contraire, elle ferait plutôt partie de ce complexe naturel de «sensations, d'attentes, d'évaluations et d'activations mémorielles et de projections» (Duranti, 2015, p. 238). On pourrait imaginer, par exemple, une activité intentionnelle sans transitivité et dans laquelle la relation à l'objet ne serait que potentielle : «chercher un voleur sans le trouver, la situation d'une vigie ou d'une sentinelle qui n'ont rien à signaler » (Descombes, 1996, p. 218). La vigie et la sentinelle sont bien là, présentes, et dont la présence n’est pas déterminée par l'existence de l'objet visé. L'intentionnalité ne serait alors qu'une «condition pour qu'une relation réelle vienne conclure un agir intentionnel» (ibid.). Husserl fait ainsi l'hypothèse d'une intentionnalité «entrelacée avec l'intersubjectivité, une propriété de notre être au monde» (Duranti, 2015, p. 238), sans désigner pour autant un objet singulier ou particulier de ce monde. L'ouverture serait à l'universel. N'est-ce pas le mode existentiel des Runa, des Korowaï, des Samoans ou d’autres sociétés pour qui, que ce soit vis-à-vis des animaux ou de leurs semblables, il existe un tissu interstitiel pré-objectif, d’avant la symbolisation, et qui prédéterminerait les relations au temps et à l'espace? La conscience symbolique ne viserait qu’un monde qui lui est déjà pré-donné.

Cependant, pour éviter de tomber dans les travers mortels du relativisme contre lesquels Husserl entendait se prémunir, dès les années 1935-1936, une forte exigence d'universalisme se fait jour dans un texte qui peut, en quelque sorte, synthétiser tout notre propos, sur un mode visionnaire : 
Si nous sommes transportés dans un milieu étranger, chez les nègres du Congo ou chez les paysans chinois, etc., nous butons alors sur le fait que leurs vérités, que les faits qui pour eux sont en général bien établis, assurés et à assurer, ne sont d'aucune façon les nôtres. Mais si nous posons comme but la vérité inconditionnellement valable pour tous sujets sur des objets, en partant de ce en quoi des Européens normaux, des Indoux normaux, des Chinois normaux, etc., malgré toute relativité, cependant finissent par s'accorder - partant donc de ce qui rend identifiables pour eux et pour nous des objets du monde de la vie communs à tout le monde, bien que la conception en demeure différente, les qualités sensibles et autres choses semblables -, alors nous parvenons quand même à trouver le chemin de la science objective. (Husserl, I976 [1954], p. I58).

Le monde de la vie, que Wittgenstein appellerait sans doute ici «les formes de vie et les jeux de langage», est-il pour autant dépassé, comme le laisse supposer ce texte? La conscience intentionnelle et symbolisante doit incontestablement faire son œuvre, mais en s'employant toujours à conserver le magma chaotique des icônes de la vie donnée avant qu'elle ne devienne donnante.

\section{BIBLIOGRAPHIE}

AFFERGAN Francis, 2012, Le Moment critique de l'Anthropologie, Paris, Hermann, «Société et Pensées».

BENJAMIN Walter, 1991 (1940), Paralipomènes et variantes des thèses «sur le concept d'histoire», in Id. Écrits français, Paris, Gallimard., p. 118-192.

BIDNEY David, 1973, «Phenomenological Method and the Anthropological Science of the Cultural Life-World», in Phenomenology and the Social Sciences, Maurice Natanson (dir.), vol.I, Norwestern University Press, Evanston, p. I09-140.

CSORDAS Thomas J., 1990, «Embodiment as a Paradigm for Anthropology», Ethos, 18, p.5-47.

- (dir.), 1994, Embodiment and Experience. The Existential Ground of Culture and Self, Cambridge Mass., Cambridge University Press.

DE MARTINO Ernesto, 1999 (1948), Le Monde magique, Paris, Institut d'édition Sanofi-Synthelabo.

-, 1999 (1959), Italie du Sud et magie, Paris, Institut d'édition Sanofi-Synthelabo. 
DESCOMBES Vincent, 1996, Les Institutions du sens, Paris, Éditions de Minuit.

DUFRENNE Mikel, 1949, «Heidegger et Kant», Revue de Métaphysique et de Morale, 54-I, p. I-28.

DURANTI Alessandro, 2015, The Anthropology of Intentions. Language in a World of Others, Cambridge, Cambridge University Press.

GRAHAM Laura, 1993, «A Public Sphere in Amazonia? The Depersonalized Collaborative Construction of Discourse in Xavante», American Ethnology, 20-4, p. 717-74l.

HENRY Michel, 1963, L'Essence de la manifestation, Paris, PUF.

-, 2000, Incarnation. Une philosophie de la chair, Paris, Seuil.

-, 2003, Phénoménologie de la vie, tome I, De la phénoménologie, Paris, PUF.

-, 2010, Pour une phénoménologie de la vie, entretien avec Olivier Salazar-Ferrer,

Clichy, Corlevour.

HUSSERL Edmund, 1950 (1913), Idées directrices pour une phénoménologie, Paris, Gallimard.

-, 1976 (1954), La Crise des sciences européennes et la phénoménologie transcendantale, Paris, Gallimard.

-, 1982 (1952), Recherches phénoménologiques pour la constitution, Paris, PUF.

-, 1996 (1928), Leçons pour une phénoménologie de la conscience intime du temps, Paris, PUF.

-, 1998 (1966), De la synthèse passive, Grenoble, Jérôme Million.

JACKSON Michael (dir.), 1996, Things As They Are. New Directions in

Phenomenological Anthropology, Bloomington, Indiana University Press.

-, 1998, Minima Ethnographica. Intersubjectivity and the Anthropological Project,

Chicago, The university of Chicago Press.

JANICAUD Dominique, 200I, Heidegger en France, tome I, Récit, Paris, Albin Michel.

KOHN Eduardo, 2013, How Forests Think. Toward an Anthropology Beyond the Human, Berkeley, Los Angeles, London, University of California Press.

Les Études philosophiques, 2012, «La méthode phénoménologique aujourd'hui», 100-I. 
MAUSS Marcel, 1968 (1934), Les Techniques du corps, in Id. Sociologie et Anthropologie, Paris, PUF, p. 363-386.

PERRET Catherine, 2013, L'Enseignement de la torture, Paris, Seuil.

RICCEUR Paul, 2013, Écrits et conférences, Tome 3. Anthropologie philosophique, Paris, Seuil.

SAINT-AUGUSTIN, 1964, Les Confessions, Joseph Trabucco (éd.), Paris, Garnier-Flammarion.

SARTRE Jean-Paul, 1943, L'Être et le Néant. Essai d'ontologie phénoménologique, Paris, Gallimard.

SEGAUD Marion, 2007, Anthropologie de l'espace. Habiter, Fonder, Distribuer, Transformer, Paris, Armand Colin.

STASCH Rupert, 2009, Society of Others. Kinship and Mourning in a West Papuan Place, Oakland, University of California Press.

-, 201I, «Ritual and Oratory Revisited: The semiotics of Effective Action», Annual Review of Anthropology, 40, p. 159-174. 